\title{
Reconstruction and Identification of Hadronic Decays of Taus using the CMS Detector
}

Michail Bachtis* (for the CMS Collaboration)

University of Wisconsin, Madison, WI 53706, USA

E-mail: bachtis@hep.wisc.edu

\begin{abstract}
New Physics beyond the Standard Model could well preferentially show up at the LHC in final states with taus. The development of efficient and accurate reconstrution and identification of taus is therefore an important item in the CMS physics programme. The potentially superior performance of a particle-flow approach can help to achieve this goal with the CMS detector. Preliminary strategies are presented in this summary for the hadronic decays of the taus.
\end{abstract}

European Physical Society Europhysics Conference on High Energy Physics, EPS-HEP 2009,

July 16 - 222009

Krakow, Poland

${ }^{*}$ Speaker. 


\section{Introduction}

Since tau is the heaviest of the three leptons, specific final states involving taus are expected to show up in the Standard Model (SM), and would appear abundantly in many processes arising from new physics beyond the SM. Taus decay to hadronic final states in about $64 \%$ of the cases. The reconstruction and the identification of these hadronic decays is therefore an essential ingredient of the CMS [1] physics programme. In this summary, the strategy used by CMS to reconstruct and identify hadronic decays of taus with particle-flow techniques is outlined. Several and substantial improvements to the tau reconstruction (through the particle-flow algorithm developments) and identification (through high-level analysis tools, like multivariate approaches) are still expected and are actively being worked on. Meanwhile, this summary represents the state of the art of the reconstruction and the basic selections. More details can be found at [2].

\section{Base Tau Identification}

Tau Identification starts from a jet reconstructed with the particle-flow algorithm [3]. The particle-flow reconstruction algorithm aims at providing a global (i.e., complete and unique) event description at the level of individually reconstructed particles, with an optimal combination of the information coming from all CMS subdetectors. The particle flow candidates can then be used for jet reconstruction and higher level identification.

The Tau-ID algorithm starts from a reconstructed particle flow jet that satisfies a minimum transverse-momentum threshold. Then the highest $p_{T}$ (leading) charged hadronic candidate(track) within a matching cone of $\Delta R<0.1$ around the jet axis is required to have $p_{T}>5 \mathrm{GeV} / c$. The boosted tau decay products are expected to be confined within a narrow cone around the leading track. Moreover an isolation annulus is defined around the signal cone where very low activity is expected for high quality isolated tau candidates. Tau isolation requires no charged candidates with $p_{T}>1.0 \mathrm{GeV} / c$ and no gamma candidates with $E_{T}>1.5 \mathrm{GeV}$ in the isolation annulus.
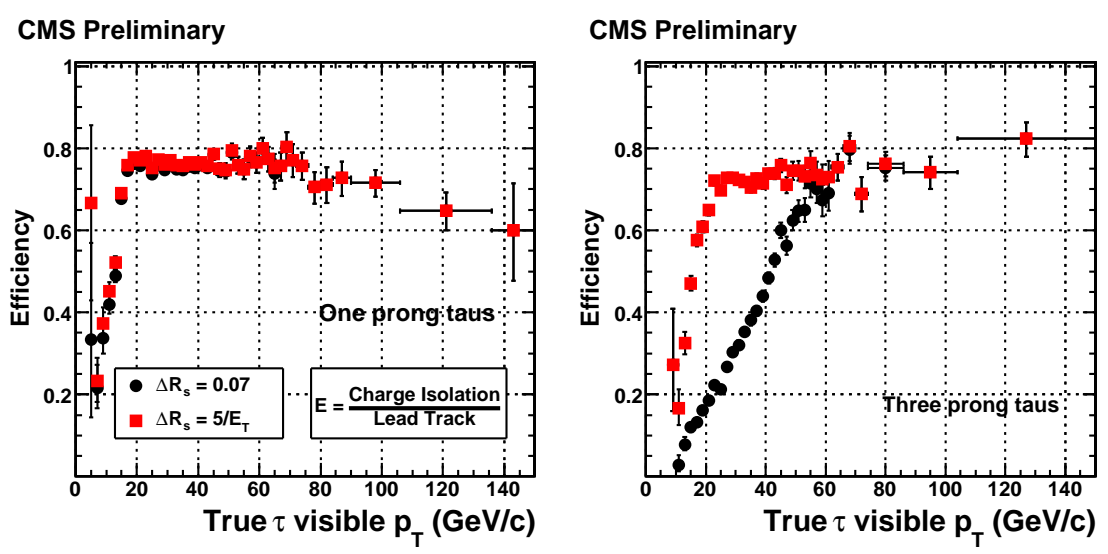

Figure 1: Comparison of charge candidate isolation efficiency for one prong (left) and three prong taus (right) for a fixed cone size of $\Delta R=0.05$ and a shrinking cone size of $5 / E_{T}$ respectively. 

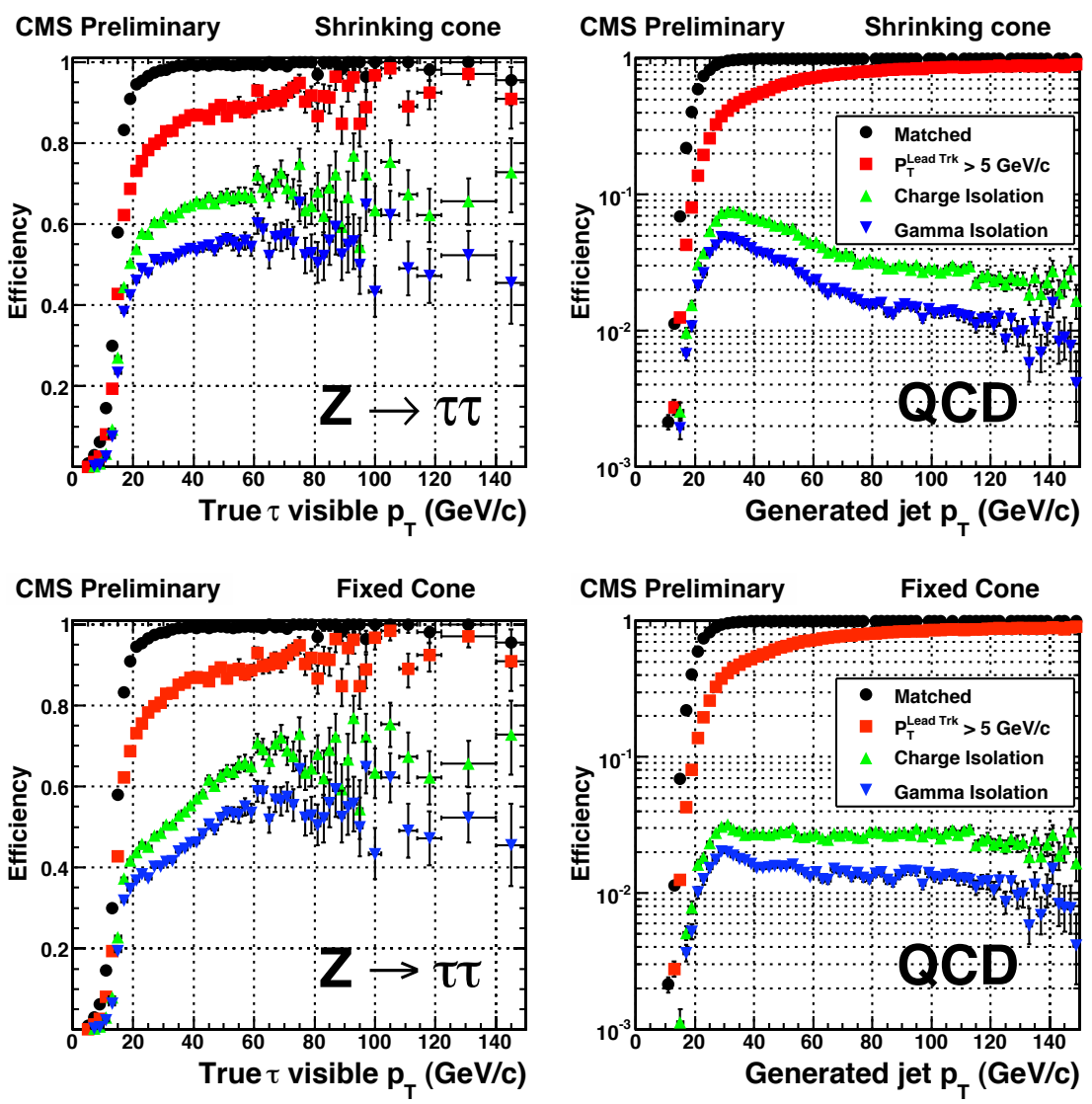

Figure 2: Performance of the base tau selection algorithm: Efficiency and Fake rate for the shrinking cone (up) and the fixed cone (down) after Particle Flow jet reconstruction, lead track finding and isolation with charged hadronic and gamma candidates

The signal cone size can be parametrized to the specific physics analysis requirements. Most of the past CMS analyses [4] were using a fixed signal cone size of $\Delta R=0.07$ and an isolation annulus of outer cone of $\Delta R=0.45$. One can exploit the fact that hadronic taus become more collimated at higher energy by requiring that the signal cone size can shrink as the transverse energy of the reconstructed jet becomes higher. Figure 1 shows track isolation efficiency for one prong and three prong taus reconstructed with a fixed cone of $\Delta R=0.07$ and a shrinking cone that scales with the jet transverse energy as $5.0 / E_{T}$ between the limits of $\Delta R=0.15$ and $\Delta R=0.07$. In the three prong case, the fixed cone of $\Delta R=0.07$ is too small to contain all three tracks and some of them are included in the isolation annulus and veto the tau candidate. An overall increase of approximately $20 \%$ in efficiency is established by using the shrinking cone. However, larger cone size at low $p_{T}$ results in lower background rejection at this region. Ongoing work is aiming at improving the background rejection by identifying the tau decay modes and using multivariate techniques. The overall performance of the algorithm is depicted in Fig. 2 for both the shrinking and fixed cone. The leading track $p_{T}$ cut provides QCD rejection up to a factor of 5 for low $p_{T}$ jets before isolation is applied. Another point of ongoing work is aiming towards reconstruction of photon conversions 
which may spoil both the energy reconstruction and the photon based isolation.

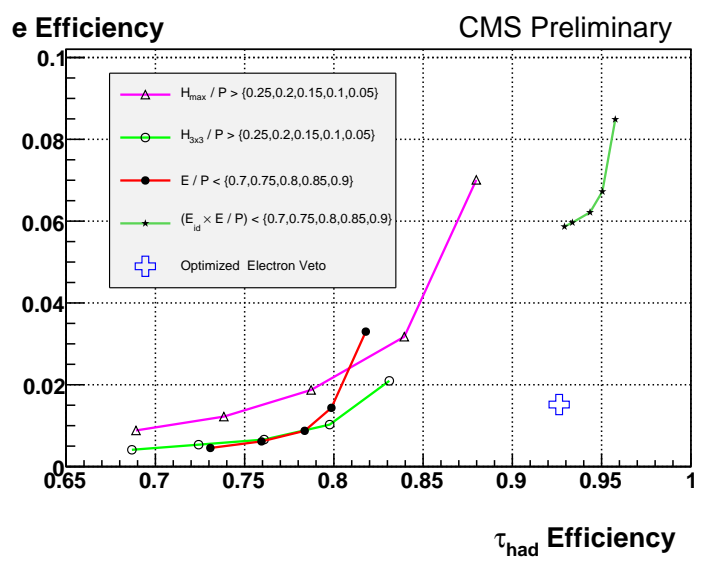

Figure 3: Performance of the relevant electron rejection cuts and the optimized electron veto

\section{Higher Level Discrimination}

After the base tau identification, higher level discriminants can be applied. Electron, muon rejection and higher level discriminants that identify the tau decay mode are already implemented.

Isolated electrons that are produced during SM Electroweak processes are often a critical source of fake taus in many physics analyses. A particle-flow electron pre-identication algorithm has been developed, described in [3] and achieves 90-95\% efficiency with about 5\% pion acceptance. To extend the electron rejection beyond $95 \%$,two additional variables are formed, which are described fully in [2]. The first variable, $E / P$, is the energy of ECAL clusters expected to contain electron bremsstrahlung photons divided by the momentum of the leading track. The second variable, $H_{3 \times 3} / P$, is the energy of HCAL clusters expected to contain the charged pion shower divided by the momentum of the leading track. Optimizing cuts on these variables separately for taus which are pre-identified as electrons as those that are not leads to an efficiency of $92.5 \%$ for true taus and $1.5 \%$ for true electrons. The electron rejection results are summarized in Fig. 3.

\section{Conclusions}

This summary describes tau reconstruction and identication using particle-flow with the CMS detector. There are three major components: a general particle flow reconstruction, a common tau reconstruction using reconstructed particles, and a higher level identication. While further signicant improvements are still being pursued, existing methods provide a strong rejection of QCD, electron and muon backgrounds, while preserving high efficiency for selecting hadronic taus.

\section{References}

[1] CMS Collaboration, The CMS experiment at the CERN LHC,JINST 3:S08004,2008 
[2] CMS Collaboration,CMS strategies for tau reconstruction and identification using particle flow techniques, CMS PAS PFT-08-001

[3] CMS Collaboration,Particle Flow based reconstruction of jets, taus and ET miss, CMS PAS PFT-09-001

[4] CMS Collaboration,Search for MSSM heavy neutral Higgs boson in two tau jets decay mode, CMS Public Note, CMS NOTE 2006/126 